"Synthesis of oligonucleotide inhibitors of DNA (Cytosine-C5) methyltransferase containing 5-azacytosine residues at specific sites.” Güimil García, R., Brank, A.S., Marquez, V.E., Christman J.K., Eritja, R. Antisense and Nucleic Acid Drug Development, 11(6), 369-378 (2001).

PMID: 11838638, doi: 10.1089/10872900175341135

\title{
SYNTHESIS OF OLIGONUCLEOTIDE INHIBITORS OF DNA (Cytosine-C5) METHYLTRANFERASE CONTAINING 5-AZACYTOSINE RESIDUES AT SPECIFIC SITES.
}
Ramon Güimil García ${ }^{1}$, Adam S. Brank ${ }^{2}$, Judith K. Christman ${ }^{2,3}$, Victor E. Marquez ${ }^{4}$, Ramon Eritja ${ }^{1,5^{*}}$

${ }^{1}$ European Molecular Biology Laboratory, Meyerhofstrasse 1, D-69117 Heidelberg. Germany;

${ }^{2}$ Department of Biochemistry and Molecular Biology and ${ }^{3}$ UNMC/Eppley Cancer Center, University of Nebraska Medical Center, Omaha, NE, 69198-4525, USA;

${ }^{4}$ Laboratory of Medicinal Chemistry, Center for Cancer Research, National Cancer Institute at Frederick, Frederick MD 21702, USA.

${ }^{5}$ Present address: Institut de Biología Molecular de Barcelona, CSIC, Jordi Girona 18-26, E-08034 Barcelona, Spain.

Corresponding author:

Ramon Eritja

Institut de Biología Molecular de Barcelona

CSIC

Jordi Girona 18-26

E-08034 Barcelona

Spain

Phone 34-93-4006145

Fax 34-93-2045904

Email: recgma@cid.csic.es

Running title: 5-azacytosine oligodeoxyribonucleotides 


\begin{abstract}
The incorporation of 5-azacytosine residues into DNA causes potent inhibition of DNA (Cytosine-C5) methyltransferases. The synthesis of oligodeoxyribonucleotides (ODNs) incorporating single or multiple 5-aza-2'-deoxycytidine residues at precise sites of the ODN was undertaken to generate an array of sequences containing the reactive 5azacytosine base at specific target sites for enzymatic methylation. Preparation of these modified oligonucleotides requires the use of 2-(p-nitrophenyl)ethyloxycarbonyl (NPEOC) groups for the protection of exocyclic amino functions. These groups are removed under mild conditions, thus avoiding conventional protocols that are detrimental to the integrity of the 5-azacytosine ring.
\end{abstract}

\title{
INTRODUCTION
}

5-Azacytosine (ZCyt or Z) is a cytosine analogue wherein the carbon at position 5 of the pyrimidine ring is replaced by a nitrogen atom. Both ribo- and 2'-deoxyribonucleosides (ZCyd and ZdCyd) versions containing this modified base are potent cytotoxic agents which have been used for the treatment of sickle cell anemia, myelodysplastic syndrome, leukemia and several other cancers with varying degrees of success (Pinto and Zagonel, 1993; Goldberg et al., 1993; Momparler et al., 1997, Rochette et al., 1994). As result of their incorporation into DNA, these drugs have a unique mechanism of action of demethylating DNA which affects gene regulation (Bender et al., 1998; Christman, 1984; Christman et al., 1985; Creusot et al., 1982). In order to help elucidate the molecular basis of the inhibitory properties of ZCyt in DNA, we have undertaken the preparation of oligodeoxyribonucleotides (ODNs) containing ZCyt. The hydrolytic instability of the triazine ring is well documented (Beisler, 1978; Lin et al. 1981) and precludes the utilization of standard phosphoramidite protocols particularly during the treatment with ammonia.

The preparation of ONDs containing the more stable 5,6-dihydro-5-azacytosine base has been described (Goddard and Marquez, 1988), but conversion of the reduced base to ZCyt was not efficient enough to yield the desired ODNs in acceptable yields (Goddard and Marquez, 1988). Previous studies (Aviñó et al., 1995) using the Hphosphonate derivative of ZdCyd without protection of the exocyclic amino group showed that the triazine ring was stable to non-nucleophilic bases (such as 1,8- 
diazabiciclo[5.4.0]undec-7-ene, DBU) in anhydrous pyridine, conditions under which the p-nitrophenylethyl (NPE, Scheme 1) and p-nitrophenylethoxycarbonyl (NPEOC, Scheme 1) groups described by Pfleiderer (Himmelsbach et al., 1984) are effectively removed (Scheme 2). Unfortunately, the H-phosphonate method was only practical for the preparation of short ODN sequences. When ODNs of 15-20 bases were prepared, the coupling efficiency of the NPEOC-protected H-phosphonate monomers dropped significantly. To overcome this problem, we decided to utilize a modified approach of the more conventional phosphoramidite methodology.

In this communication, we describe the preparation of the NPEOC-protected phosphoramidite of ZdCyd and its use in the preparation of longer ONDs containing ZCyt. A short description of this work was previously reported (Eritja et al., 1997).

\section{MATERIALS AND METHODS}

2'-Deoxy-N $N^{4}$-[2-(4-nitrophenyl)ethoxycarbonyl]-5-azacytidine (2).

2'-Deoxy-5-azacytidine (228 mg, $1 \mathrm{mmol}$ ) was dried after three successive evaporations of a solution in anhydrous pyridine. The residue was dissolved in anhydrous DMF and treated with $0.53 \mathrm{ml}$ (2.5 mmol) of hexamethyldisilazane. After stirring $1 \mathrm{~h}$ at room temperature the solution was concentrated, and the residue was dried by three cycles of evaporation from toluene ( $5 \mathrm{ml}$ ) yielding a white foam (TLC, Rf 0.66, 20\% ethanol in dichloromethane). The residue was then thrice dissolved in $5 \mathrm{ml}$ of anhydrous pyridine and concentrated to dryness, before finally dissolving it in $30 \mathrm{ml}$ of anhydrous pyridine. To this solution, $350 \mathrm{mg}$ (1.5 mmol) of 2-(p-nitrophenyl)ethyl chloroformate dissolved in $5 \mathrm{ml}$ of dichloromethane was added, and after $30 \mathrm{~min}$ of magnetic stirring at room temperature the reaction was judged complete by TLC ( $\mathrm{Rf} 0.93,10 \%$ ethanol in dichloromethane). The solution was then concentrated to dryness, and traces of pyridine were removed by three cycles of evaporation of a toluene solution $(5 \mathrm{ml})$. The residue was dissolved in chloroform and the organic solution was washed with $1 \mathrm{M}$ sodium bicarbonate, dried $\left(\mathrm{Na}_{2} \mathrm{SO}_{4}\right)$ and concentrated to dryness.

Removal of the trimethylsilyl groups in DMF solutions. The residue from the above reaction was dissolved in DMF/ THF (3:1), and the resulting solution was stirred for 3 days at room temperature. After the solvents were removed, the residue was triturated with ether to form a white solid that was purified by column chromatography on silica gel ( 3 to $10 \%$ step gradient of ethanol in chloroform) to give $180 \mathrm{mg}(0.43 \mathrm{mmol}, 43 \%)$ of the 
desylilated product. [TLC (10\% ethanol in chloroform) $\mathrm{Rf}=0.53$; (5\% ethanol in chloroform) Rf $=0.12$ ]. ${ }^{1} \mathrm{H}-\mathrm{NMR}$ (DMSO-d $\left.{ }_{6}\right) \delta(\mathrm{ppm}): 10.3$ (wide s, $1 \mathrm{H}, \mathrm{NH}$ ), 8.41 (s, 1H, H-6), 7.71 (d, 2H, J= 8.6 Hz, NPEOC), 7.18 (d, 2H, J= $8.6 \mathrm{~Hz}, \mathrm{NPEOC}$ ), 5.56 (t, 1H, H-1'), 4.86 (d, 1H, 3'-OH), 4.71 (t, 1H, 5'-OH), 3.92 (t, 2H, CH $\left.\mathrm{CH}_{2} \mathrm{NPOC}\right) 3.8$ (m, 1H, H3'), 3.45 (m, 1H, H-4'), 3.17 (m, 2H, H-5'), 2.64 (t, 2H, $\left.\mathrm{CH}_{2} \mathrm{NPEOC}\right), 1.8$ (m, 2H, H-2'). ${ }^{13} \mathrm{C}-\mathrm{NMR}\left(\mathrm{CDCl}_{3}\right) \delta$ (ppm): 163.0 (C-4), 157.1 (C-6), 153.5 (CO, NPEOC), 150.7 (C-2), 146.7 (C-NO ${ }_{2}$ ), 145.5 (Cq, Ar), 129.9 (CH, Ar), 123.5 (CH, Ar), 87.9 (C-4'), 86.7 (C-1'), 67.7 (C-3'), $65.4\left(\mathrm{CH}_{2}\right), 40.4\left(\mathrm{C}-2\right.$ '), $34.6\left(\mathrm{CH}_{2}\right)$. Anal. Calcd. for $\mathrm{C}_{17} \mathrm{H}_{19} \mathrm{~N}_{5} \mathrm{O}_{8}$ (Found: $\mathrm{C}$, 48.1\%; H, 4.7\%; N, 16.4\%; requires C, 48.5\%; H, 4.6\%; N, 16.6\%).

Removal of the trimethylsilyl groups with TAS-F. Alternatively, the silyl protected product resulting from a $4 \mathrm{mmol}$ scale reaction was treated with a solution of tris(dimethylamino) sulfonium difluorotrimethylsilicate (TAS-F) (12 mmol, $3.3 \mathrm{mmol}$ ) in DMF (50 ml). After $2 \mathrm{hrs}$ at room temperature, the solution was concentrated to dryness and the residue was purified by column chromatography on silica gel as described above to give the same product as before (1.35 g, $3.2 \mathrm{mmol}, 80 \%)$.

\section{2'-Deoxy-5'-O-dimethoxytrityl- $N^{4}$-[2-(4-nitrophenyl)ethoxycarbonyl]-5-azacytidine (3).}

2'-Deoxy- $\mathrm{N}^{4}$-[2-(4-nitrophenyl)ethoxycarbonyl]-5-azacytidine (1.63 g, $3.87 \mathrm{mmol}$ ) was dried after repeated evaporation of a solution in anhydrous pyridine and reacted with dimethoxytrityl chloride (1.44 g, $4.26 \mathrm{mmol})$ in pyridine $(20 \mathrm{ml})$. After 3 hrs stirring at room temperature, the solution was concentrated to dryness. Traces of pyridine were removed by three cycles of evaporation of a toluene solution $(5 \mathrm{ml})$. The residue was dissolved in dichloromethane, and the organic solution was washed with $1 \mathrm{M}$ sodium bicarbonate aqueous solution, dried $\left(\mathrm{Na}_{2} \mathrm{SO}_{4}\right)$ and concentrated to dryness. The residue was purified by column chromatography on silica gel eluted with a 0 to $5 \%$ step gradient of ethanol in chloroform to give $2.01 \mathrm{~g}$ ( $2.78 \mathrm{mmol}, 71 \%)$ of 3 . [TLC (10\% ethanol in dichloromethane) $\mathrm{Rf}=0.40]$. IR $\left(\mathrm{cm}^{-1}\right)$ : 3400, 2960, 1770, 1700, 1610, 1510, 1350, 1250, 1220, 1180, 1030, 830. ${ }^{1} \mathrm{H}-\mathrm{NMR}\left(\mathrm{CDCl}_{3}\right) \delta$ (ppm): 8.60 (s, 1H, H-6), 8.16 (d, 2H, Ar, NPEOC) 7.43 (d, 2H, Ar, NPEOC), 7.1-7.3 (m, 9H, Ar, DMT), 6.81 (d, 4H, Ar, DMT), 6.16 (t, 1H, H-1'), 4.4 (m, 3H, H-3', $\mathrm{CH}_{2}$ NPEOC), 4.2 (m, 1H, H-4'), 3.77 (s, 6H, Me

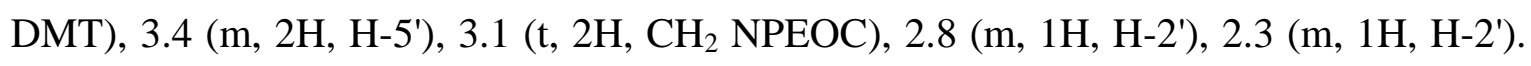

${ }^{13} \mathrm{C}-\mathrm{NMR}\left(\mathrm{CDCl}_{3}\right) \delta$ (ppm): 163.1 (C-4), 158.4 (Cq, DMT), 156.8 (C-6), 153.4 (CO, NPEOC), 150.2 (C-2), 146.7 (C-NO $), 145.1$ (Cq, Ar), 144.2 (Cq, DMT), 135.4 (Cq, DMT), 135.2 (Cq, DMT), 129.7 (CH, Ar), 127.8 (CH, DMT), 126.7 (CH DMT), 123.5 
(CH, Ar), 113.1 (CH Ar), 87.7 (C-4'), 86.9 (C-1'), 86.8 (Cq, DMT), 71.2 (C-3'), 65.5 $\left(\mathrm{CH}_{2}\right), 63.1(\mathrm{C}-5)$ ') $55.0\left(\mathrm{CH}_{3}\right), 41.9\left(\mathrm{C}-2^{\prime}\right), 34.5\left(\mathrm{CH}_{2}\right)$. NOTE: It would have been nice to have a better characterization of this compound (Anal. or MS).

\section{N,N-Diisopropylamino-2-(p-nitrophenyl)ethoxy chlorophosphine.}

In a 1-liter round bottom flask, $17.5 \mathrm{ml}(200 \mathrm{mmol})$ of phosphorous trichloride was dissolved in $500 \mathrm{ml}$ of anhydrous acetonitrile and kept under argon atmosphere. The solution was cooled over an ice-bath and treated dropwise with a solution of 2-( $p$ nitrophenyl)ethanol (3.34 g, $20 \mathrm{mmol}$ ) dissolved in $100 \mathrm{ml}$ of acetonitrile. The reaction mixture was gradually warmed to room temperature and stirred for 1 hour. The mixture was concentrated to give a yellowish oil that was used in the next step without further purification. The crude 2-(p-nitrophenyl)ethoxy dichlorophosphine obtained was dissolved in $500 \mathrm{ml}$ of acetonitrile, and the solution was cooled with an ice-bath. To this solution $6.2 \mathrm{ml}$ (44 mmol) of $\mathrm{N}, \mathrm{N}$-diisopropylamine dissolved in $100 \mathrm{ml}$ of acetonitrile was added, and after the addition the mixture was gradually warmed to room temperature after 6 hours of stirring. The white precipitate was filtered and the filtrate was concentrated to dryness to give $5 \mathrm{~g}$ (15 mmol, 75\%) of a yellowish oil that was used directly in the preparation of all phosphoramidites without further purification. ${ }^{31} \mathrm{P}-\mathrm{RMN}$ $\delta_{\mathrm{P}}\left(75 \mathrm{MHz}, \mathrm{CDCl}_{3}\right): 177.74 \mathrm{ppm}$ (90\% pure by phosphorous NMR).

Synthesis of the 2-(p-nitrophenyl)ethyl phosphoramidites of the natural bases and 5methyl-2'-deoxycytidine.

The appropriate 5'-DMT-N,O-(NPEOC, NPE)-protected 2'-deoxynucleoside (2 mmol) was dried after several coevaporations with dry acetonitrile. The residue was dissolved in $50 \mathrm{ml}$ of anhydrous tetrahydrofuran containing $1 \mathrm{ml}(6 \mathrm{mmol})$ of diisopropylethylamine. The solution was cooled over an ice-bath and $1.0 \mathrm{~g}$ (3 mmol) of N,N-diisopropylamino-2-( -nitrophenyl)ethoxy chlorophosphine was added. After $1 \mathrm{hr}$ of magnetic stirring at room temperature, the solution was concentrated to dryness. The residue was dissolved in dichloromethane and the organic solution was washed with $1 \mathrm{M}$ sodium bicarbonate and saturated sodium chloride solutions, dried $\left(\mathrm{Na}_{2} \mathrm{SO}_{4}\right)$ and concentrated to dryness. The residue was purified by column chromatography on silica gel with ethyl acetate / dichloromethane (1:1). The column was packed with the solvent mixture containing $1 \%$ triethylamine and eluted with the mixture without triethylamine. The appropriate fractions were collected and concentrated to dryness. 
3'-O-NPE phosphoramidite of DMT-T: Yield: 83\% (1.41g, $1.67 \mathrm{mmol})$. TLC (ethyl acetate / dichloromethane 1:1) $\mathrm{Rf}=0.85,{ }^{31} \mathrm{P}-\mathrm{RMN} \delta_{\mathrm{P}}\left(75 \mathrm{MHz}, \mathrm{CDCl}_{3}\right): 144.05,144.43$ ppm.

3'-O-NPE phosphoramidite of DMT-C(NPEOC): Yield: 76\% (1.55g, $1.52 \mathrm{mmol})$. TLC (ethyl acetate / dichloromethane 1:1) Rf $=0.78,{ }^{31} \mathrm{P}-\mathrm{RMN} \delta_{\mathrm{P}}\left(75 \mathrm{MHz}, \mathrm{CDCl}_{3}\right): 144.62$ ppm.

3'-O-NPE phosphoramidite of DMT-G(NPE, NPEOC): Yield: 79\% (1.90g, $1.52 \mathrm{mmol})$. TLC (ethyl acetate / dichloromethane 1:1) Rf $=0.93,{ }^{31} \mathrm{P}-\mathrm{RMN} \delta_{\mathrm{P}}\left(75 \mathrm{MHz}, \mathrm{CDCl}_{3}\right)$ : $143.98 \mathrm{ppm}$.

3'-O-NPE phosphoramidite of DMT-A(NPEOC): Yield: 77\% (1.61g, $1.54 \mathrm{mmol})$. TLC (ethyl acetate / dichloromethane 1:1) Rf $=0.85,{ }^{31} \mathrm{P}-\mathrm{RMN} \delta_{\mathrm{P}}\left(75 \mathrm{MHz}, \mathrm{CDCl}_{3}\right)$ : 143.07, $143.37 \mathrm{ppm}$.

3'-O-NPE phosphoramidite of DMT-5-methyl-C(NPEOC): Yield: 83\% (0.79g, 0.76 mmol, starting from $0.92 \mathrm{mmol}$ ). TLC (ethyl acetate / dichloromethane 1:1) Rf =0.89, ${ }^{31} \mathrm{P}-\mathrm{RMN} \delta_{\mathrm{P}}\left(75 \mathrm{MHz}, \mathrm{CDCl}_{3}\right): 144.42 \mathrm{ppm}$

2'-Deoxy-5'-O-dimethoxytrityl- $N^{4}$-[2-(4-nitrophenyl)ethoxycarbonyl]-5-azacytidine 3'-O(2-(p-nitrophenyl)ethoxy)- $N, N$-diisopropyl phosphoramidite (4).

2'-Deoxy-5'-O-dimethoxytrityl-N ${ }^{4}$-[2-(4-nitrophenyl)ethoxycarbonyl]-5-azacytidine ( $1 \mathrm{~g}, 1.38 \mathrm{mmol}$ ) was dissolved in anhydrous tetrahydrofuran $(35 \mathrm{ml})$ containing $0.69 \mathrm{ml}$ (4.14 mmol) of diisopropylethylamine. The solution was cooled in an ice-bath and 2.04 mmol of N,N-diisopropylamino-2-(p-nitrophenyl)ethoxy chlorophosphine was added. After $1 \mathrm{hr}$ of magnetic stirring at room temperature, the solution was concentrated to dryness. The residue was dissolved in dichloromethane and the organic solution was washed with $1 \mathrm{M}$ sodium bicarbonate and saturated sodium chloride solutions, dried $\left(\mathrm{Na}_{2} \mathrm{SO}_{4}\right)$ and concentrated to dryness. The residue was purified by column chromatography on silica gel with ethyl acetate / hexane (2:1). The column was packed with the solvent mixture containing $1 \%$ triethylamine and eluted with the same solvent 
mixture without triethylamine. Yield: 78\% (1.0g, $1.08 \mathrm{mmol})$. TLC (ethyl acetate / hexane 2:1) $\mathrm{Rf}=0.64$, (5\% etanol in dichloromethane $+1 \%$ triethylamine) $\mathrm{Rf}=0.93$. ${ }^{31} \mathrm{P}-\mathrm{RMN} \delta_{\mathrm{P}}\left(75 \mathrm{MHz}, \mathrm{CDCl}_{3}\right): 145.7 \mathrm{ppm}$.

Oligodeoxynucleotid e (ODN) syntheses.

ONDs were prepared in a $1 \mu \mathrm{mol}$ scale using phosphoramidite 4 and the 2-(4nitrophenyl)ethyl phosphoramidites of the natural bases protected with the NPE and NPEOC groups. Controlled-pore glass supports having the NPE,NPEOC-protected nucleosides attached through an oxalyl linkage were used (Alul et al., 1991; Aviñó et al., 1996). Some phosphoramidites were not very soluble in acetonitrile and were dissolved in dry dichloromethane to obtain $0.1 \mathrm{M}$ solutions. Standard cycles were used with a slight modification of coupling times which were increased from $30 \mathrm{sec}$ to $2 \mathrm{~min}$. Coupling efficiencies were $>96 \%$ as measured by DMT analysis. Deprotection was carried out by treatment of the ODN-supports with a 0.5 M DBU solution in pyridine containing $5 \mathrm{mg}$ of thymine for $15 \mathrm{hrs}$ at room temperature. The resulting solutions were neutralized with acetic acid and concentrated to dryness. The residues were dissolved in water and desalted with a Sephadex G-25 or G-10 column.

Sequences I-V (see Table 1) were prepared with the last DMT group on. Purification of these ONDs was performed by HPLC using the following solutions: Solvent A: 5\% ACN in $100 \mathrm{mM}$ triethylammonium acetate (pH 6.5) and solvent $\mathrm{B}: 70 \%$ ACN in $100 \mathrm{mM}$ triethylammonium acetate $\mathrm{pH}$ 6.5. Columns: PRP-1 (Hamilton), $250 \mathrm{x}$ $10 \mathrm{~mm}$. Flow rate: $3 \mathrm{ml} / \mathrm{min}$ and a 30 min linear gradient from $10-80 \% \mathrm{~B}$. Yield (OD units at $260 \mathrm{~nm}$ after HPLC purification: DMT-heptamer I, 2.2 OD (45 $\mu \mathrm{g})$; DMToctamer II, 6.3 OD (0.18 mg); DMT-15mer III, 15 OD (0.45 mg); DMT-24mer IV, 25 OD (0.75 mg); and DMT-24mer V, 2.3 OD (80 $\mu \mathrm{g})$. DMT-ONDs I-V were treated with 1 $\mathrm{ml}$ of acetic acid for $30 \mathrm{~min}$ at room temperature. The acetic acid was removed by extractions with ether and the ODNs without the DMT group were purified by HPLC using similar conditions as before but with a 30 min linear gradient from $0-50 \%$ B. Yield

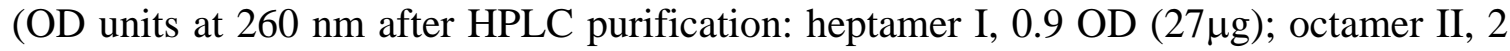
OD (60 $\mu \mathrm{g})$; 15mer III, 6 OD (0.18 mg); 24mer IV, 7.5 OD (0.22 mg); and 24mer V, 0.8 OD (24 $\mu \mathrm{g})$.

ODN sequences III, V-XV (see Table 2) were prepared removing the last DMT group on the synthesizer and purified by HPLC. Yield (OD units at $260 \mathrm{~nm}$ after HPLC purification): OND III, 15 OD (0.45 mg); ODN V, 10 OD (0.3 mg); OND VI, 8 OD (0.24 
mg); ODN VII, 14 OD (0.42 mg); ODN VIII, 5 OD (0.15 mg); ODN IX, 9 OD (0.27 mg); ODN XI, 27 OD (0.81 mg); ODN XII, 15 OD (0.45 mg); ODN XIII, 3 OD ( $90 \mu g)$; ODN XIV, 45 OD (1.35 mg); and ODN XV, 55 OD (1.65 mg). Five independent syntheses ( $1 \mu \mathrm{mol}$ each) of sequence $\mathrm{X}$ were performed yielding, respectively, 24, 35, 45, 42 and 21 OD. (NOTE: I changed this for consistency with the previous paragraph)

Selected ODNs were characterized by mass spectrometry using electrospray ionization under neutral conditions. Heptamer I (5' TAGZTGA 3') : expected 2120, found $2120.4(\mathrm{M})$ in addition to peaks at 2110.5 (M-10) and 1830.8 (M-290) plus sodium adducts. Octamer II (5’ TAZGZTGA 3') : expected 2643, found 2642.4 (M) in addition to a peak at $2632.4(\mathrm{M}-10)$ plus sodium adducts. Fifteenmer III: (5' GCAATGGAZCCTCTA 3') expected 4537, found 4537 (M), in addition to peaks at 4527 (M-10) and 4236 (M-290) plus sodium adducts. Sequence of 15mer III carrying the DMT group (5’ DMT-GCAATGGAZCCTCTA 3') : expected 4840, found 4839.3 (M), with no M-10 and M-290 peaks observed. Characterization of 24mer ODNs using MALDI-TOF was not possible because of fragmentation during acquisition.

Characterization of oligonucleotides (ODNs) by gel electrophoresis.

ZCyt-ODN XII and the corresponding ODN containing cytosine instead of ZCyt were enzymatically radiolabeled with ${ }^{32} \mathrm{P}$ at the 5 '-end using $\mathrm{T} 4$ kinase and $\gamma{ }^{32} \mathrm{P}$-ATP [Sambrook et al. 1980]. These ODNs were suspended in TE (10 mM Tris-HCl, pH 7.8, 1 mM EDTA) and analyzed directly (ss-ZCyt-ODN) or as annealed duplexes (obtained after incubation at $60{ }^{\circ} \mathrm{C}$ for $60 \mathrm{~min}$, followed by slow cooling to $37{ }^{\circ} \mathrm{C}$ ) with their complementary strands. Twenty ng of ss-ODNs or $40 \mathrm{ng}$ ds-ODNs were suspended in 10 $\mu \mathrm{L}$ of distilled (twice) water and mixed with $40 \mu \mathrm{L}$ glacial acetic acid followed by $60 \mathrm{~min}$ incubation at $37^{\circ}$ for $60 \mathrm{~min}$. For HhaI digestion, $20 \mathrm{ng}$ ss-Cyt-ODN or $40 \mathrm{ng}$ ds-CytODN XII were incubated at $37^{\circ} \mathrm{C}$ for 60 min with $60 \mathrm{U}$ of $\mathrm{HhaI}$ in $50 \mu \mathrm{L}$ NEBuffer 4 (New England Biolabs, Beverley, MA). Samples in glacial acetic acid were vacuum dried in a Savant Speed-Vac and resuspended in $10 \mu \mathrm{L}$ distilled water containing $0.25 \%$ xylene cyanol, $0.25 \%$ bromphenol blue and $30 \%$ glycerol. All other samples were brought to the same final concentrations of tracking dyes and glycerol prior to electrophoresis. 8M urea$20 \%$ acrylamide gels were formed and pre-electophoresed for $30 \mathrm{~min}$ at 200V in TBE (90 $\mathrm{mM}$ Tris-Borate, $\mathrm{pH}$ 8.0, 2 mM EDTA). Samples were also electrophoresed at 200V for 30 min or until the tracking dye reached the end of the gel. Radiolabeled ODNs in 
vacuum dried gels were detected and quantified by PhosphorImager (Molecular Dynamics) analysis.

\section{Melting experiments.}

Equimolar solutions of each complementary pentadecamer (Table 3) dissolved in $50 \mathrm{mM}$ Tris-HCl pH 7.5, $0.15 \mathrm{M} \mathrm{NaCl}$ were mixed. The resulting solutions were heated to $90{ }^{\circ} \mathrm{C}$, allowed to cool slowly to room temperature and then samples were kept in the refrigerator overnight. UV absorption spectra and melting experiments (absorbance vs temperature) were recorded in $1 \mathrm{~cm}$ path-length cells using a spectrophotometer equipped with a temperature controller with a programmed step increase of $0.5{ }^{\circ} \mathrm{C} / \mathrm{min}$. Melting curves were measured at $260 \mathrm{~nm}$ on duplex concentration of $4 \mu \mathrm{M}$.

\section{RESULTS AND DISCUSSION}

Preparation of the NPEOC-protected phosphoramidite of ZCyd.

We first attempted to prepare the phosphoramidite derivative of 5'-O-dimethoxytritylZdCyd without protection of the exocyclic amino group. The product was obtained in moderate yields; however, coupling of this compound to a growing ODN chain using standard phosphoramidite conditions resulted in severe branching after the addition of the ZdCyd monomer (data not shown). For this reason, protection of the exocyclic amino group was judged necessary and the NPEOC group was selected for this purpose. ZdCyd was prepared as described (Piskala and Sorm, 1978), and the introduction of the NPEOC at the exocyclic position was performed by the transient protection method ( $\mathrm{Ti}$ et al., 1982) (Scheme 3). First, reaction of ZdCyd (1) with hexamethyldisilazane produced the silylated nucleoside which was treated with 2-(4-nitrophenyl)ethoxycarbonyl chloroformate to give the $\mathrm{N}^{4}$-NPEOC-derivative of 3',5'-O-bis(trimethylsilyl)-ZdCyd. The removal of the trimethylsilyl groups without destroying the base was the critical step on this approach. Use of tetrabutylammonium fluoride, ammonium fluoride, sodium ethoxide, $p$-toluensulfonic acid or acetic acid effectively removed the silyl groups, but also caused the breakdown of the base. However, a gradual but complete removal of the silyl groups without detectable side reactions was achieved after maintaining the solution of the silylated nucleoside in DMF for 4 days at room temperature. Using this method, the NPEOC-protected nucleoside (2) was obtained in 43\% yield after silica gel 
purification. Finally, it was discovered that the trimethylsilyl groups could be removed more efficiently using tris(dimethylamino) sulfonium difluorotrimethylsilicate (TAS-F) (Scheidt et al., 1998). This source of fluoride was able to effectively remove the trimethylsilyl groups and the desired product $\mathbf{2}$ was obtained in $80 \%$ yield after silica gel purification.

Reaction of $\mathbf{2}$ with DMT-Cl followed by reaction with the appropriate chlorophosphine gave the desired phosphoramidite (4) in good yields. During all these reactions, special care was taken to eliminate traces of pyridine before any aqueous work-up. Failure to do so resulted in complete degradation of the nucleoside.

The rest of the phosphoramidites corresponding to the natural bases protected with the NPE and NPEOC groups (Scheme 4) were prepared following previously described protocols (Himmelsbach et al., 1984; Aviñó and Eritja, 1994; Díaz et al., 1997), including the NPE-phosphoramidite of 5-methyl-2'deoxycytidine (M) protected with the NPEOC group (Ferrer et al., 1996).

Synthesis of the oligodeoxyribonucleotides (ODNs) containing 5-azacytosine (ZCyt).

ODN sequences ranging from 7 to 24 nucleotides (I-XV, Tables 1 and 2) were prepared. Heptamer I and octamer II are two small sequences that were used for full characterization and detailed analysis. Pentadecamer III and mismatched variants (Table 3) were synthesized to study the hybridization properties and stability of the ODNs carrying ZCyt. ODN sequences with 24 bases (IV-IX, XII-XV) were designed to contain target sequences for DNA (Cytosine-C5) methyltransferases. Comparable sequences had been used previously for the characterization of the inhibitory properties of ODNs containing 5,6-dihydro-5-azacytosine (Marquez et al., 1999; Sheikhnejad et al., 1999). Finally, 13mers X and XI, with similar target sequences for methylation, were prepared for future co-crystallization studies with the M.HhaI, a bacterial DNA (Cytosine-C5) methyltransferase. All ODNs were synthesized in $1 \mu \mathrm{mol}$ scale using phosphoramidite 4 and the NPE phosphoramidites of the natural bases protected with the NPE and NPEOC groups (Scheme 4). Controlled-pore glass (CPG) supports having the NPE, NPEOCprotected nucleosides attached through an oxalyl linkage were used (Alul et al., 1991). The oxalyl bond is efficiently cleaved by DBU solutions with higher efficiency (Aviñó et al., 1996) than the previously described NPE linkage (Eritja et al., 1992). Coupling efficiencies judged by the absorbance of the DMT cation released during detritylation were greater than $96 \%$. 
Initially, syntheses of sequences I-V were performed. To facilitate purification the last DMT group on the ODN was left on. Deprotection was carried out by treatment of ODN-supports with a $0.5 \mathrm{M}$ DBU solution in pyridine containing $5 \mathrm{mg}$ of thymine for 15 hours at room temperature (Aviñó and Eritja, 1994; Aviñó et al., 1996). Purification of the final products was performed by HPLC using the standard DMT-on and DMT-off protocols. HPLC chromatograms were similar to those of unmodified ODNs but yields were lower and variable (0.8-7.5 O.D. units, see Table 1). For comparison, one may expect about 30-40 OD units for a 20mer in a $1 \mu$ mol synthesis. Lower yields could be attributed to a less efficient cleavage from the linkage ODN-support (Aviñó et al., 1996), or to the observed drop ( $50 \%$ or more) in the recovery of product after removal of the DMT with acetic acid (Table 1). This loss of product was initially thought to result from the instability of the 5-azacytosine ring during acetic acid treatment to remove the DMT group. However, no degradation products (shorter ODNs) were detected by gel electrophoresis after treatment with acetic acid (vide infra).

Characterization of the products obtained was attempted by enzymatic digestion and mass spectrometry. Enzymatic digestion (NOTE: Specify what enzyme was used for this digestion and some description of the process should be included in the Experimental Section) was used first to characterize ODNs I and II. After enzyme treatment no ZdCyd was detected, even though ODNs I and II do not contain any dC that could co-elute with ZdCyd and mask its presence. Thus, it appeared that the most probable cause for the absence of detectable ZCyd was nucleoside degradation during incubation and enzymatic digestion at pH 8.5 (Beisler, 1978, Lin et al., 1981).

Electrospray ionization mass spectrometry analysis under neutral conditions gave the expected molecular weights for ODNs I-III, thus confirming both the integrity of the ODN and that indeed degradation of 5-aza-2'-deoxycytidine happened during the enzymatic digestion. Special care was taken to avoid acids or bases during the acquisition of the spectra, which could cause the degradation of the sensitive base. Although electrospray ionization under neutral conditions gave clear molecular ion peaks for ODNs I-III, along with the peaks corresponding to the sodium adducts, it failed to give the expected molecular ion peaks for the 24mers. In addition to the expected molecular weights, analysis of purified ODNs I-III also showed the presence of small amounts of two other components: one having $10 \mathrm{amu}$ units (or Daltons) less than the expected mass (M-10) and a second unknown peak 290 amu units less (M-290). The M10 product was presumed to result from the opening of the hydrated ZCyt ring with 
subsequent loss of a formyl group (Scheme 5). Such spontaneous ring opening and loss of a formyl group has been observed in ZCyt derivatives (Beisler, 1978; Lin et al., 1981) and in 5-aza-2'-deoxycytidine-thymine dimers (Goddard and Marquez, 1989). The M290 peak, on the other hand, is difficult to explain since it corresponds to an ODN that is missing a dideoxydidehydro-5-azacytidine phosphate unit $\left(\mathrm{C}_{8} \mathrm{H}_{11} \mathrm{~N}_{4} \mathrm{O}_{6} \mathrm{P}\right)$ altogether! Since coupling yields with 5-aza-2'-deoxycytidine phosphoramidite were high, and the capping step should have prevented the elongation of unreacted sequences, the M-10 and M-290 products could have resulted from the degradation of the unstable ZdCyd ring opening either under oxidation or detritylation conditions during ODN synthesis. However, mass spectrometric analysis of the DMT-oligonucleotide III showed the absence of M-10 and M-290 peaks, and only following the removal of the DMT with acetic acid and ion exchange HPLC purification these peaks were observed. Although this two-step protocol led to the enrichment of the impurities relative to the desired product, we can only explain the origin of the M-10 peak. (NOTE: The M-290 peak implies that a dideoxydidehydro-5-azacytidine phosphate unit was plucked out of the middle of the strand and the ODN was reconnected. That is hard to postulate specially in view of the lack of cleavage observed in gels after acetic acid treatment. I think Judy has a good point here and we should simply say that we don't understand). Characterization of 24-mers ODNs was not possible using MALDI-TOF because the larger ODNs fragmented during the spectral adquisition.

Stability of ODNs III and XII was monitored by gel electrophoresis (Figures 1 and 2). This technique confirmed that ZCyt-ODNs were stable in water in neutral $\mathrm{pH}$ (for at least 48 hours). Overnight treatment with concentrated ammonia at $55{ }^{\circ} \mathrm{C}$ caused complete breakdown of the ODNs into two shorter sequences, as would be expected if scission occurred at ZCyt residue (Figure 1). Products coming from the acetic acid treatment did not stain with ethidium bromide (Figure 1). Therefore, to obtain a better picture of the effect of acetic acid on ZCyt-ODNs, 5'- ${ }^{32} \mathrm{P}$-radiolabeled single stranded (ss) ODNs and double stranded (ds) ODNs formed after annealing with the complementary strand were incubated in $80 \%$ acetic acid at $37^{\circ}$ for $30-60$ min and analyzed by electrophoresis on $8 \mathrm{M}$ urea-20\% polyacrylamide gels. The result of a typical treatment is shown in Figure 2. The mobility of the acetic acid treated ODN XII (Lanes 1-3) was compared with that of an ODN of identical sequence but with cytosine replacing ZCyt (Cyt-oligonucleotide), before (Lanes 4,5) or after cleavage with HhaI (Lane 6). The Cyt-ODN treated with HhaI serves as a control for any breakdown of a 
standard ODN during acetic acid treatment. Since the ZCyt in ODN XII is in the recognition/cleavage site for HhaI, the cleaved Cyt-ODN serves as a molecular marker for an ODN 8 bases long. As can be seen, whether treated as a single-strand (ss) ODN or after annealing with a complementary ODN to form a double stranded (ds) ODN, little general breakdown of full-length ODN XII occurs after a 60 min treatment with acetic acid. Only a small proportion of the ODN appears to be converted to fragments of a size (6 bases long) expected for scission at the ZCyt residue (compare Lanes 2, 3 with Lane 6). These results suggest that even if opening of the ZCyt ring occurs, decomposition to a form that results in the scission of the phosphodiester bond does not occur rapidly under acidic conditions. These results are in contrast with the reported instability of ZCyd and ZdCyd (Beisler, 1978; Lin et al., 1981), which leads us to conclude that ZCyt is more stable inside the ODN polymer than as a free nucleoside. Although it can be argued that the direct decomposition of the base could not be detected by gel analysis, the results obtained with the ammonia treatment indicate that we could detect the breakdown at the ODN abasic DNA site resulting from the elimination of ZCyt (Scheme 5).

Due to the poor recovery of ODN containing ZCyt after treatment with acetic acid, ODN sequences III and VI-XV were prepared without the last DMT group and purified directly using reversed-HPLC DMT-off conditions (Table 2). Purity of the ODNs was monitored by gel electrophoresis and in all cases they appeared to be homogeneous. Three oligonucleotides (XIII, XIV and XV) also contained 5-methyl-cytosine (M). In these cases, the appropriate monomer carrying the NPEOC group was utilized.

A strong correlation between yield of ODN and the nature of the nucleoside present at the 3'-end was observed (Table 2). Thus, ODNs V, VI, VII, VIII, IX, XII and XIII, all of which have pyrimidines at the 3'-end were obtained in poorer yields (3-15 OD units). ODNs having purines at the 3'-end, on the other hand, were obtained in much better yields: 24-45 OD units of ODN sequences having a G at the 3'-end (X and XII) and 1555 OD units of oligonucleotides having $A$ at the 3'-end (III, XIV and XV). The proposed mechanism of cleavage for the oxalyl bond is a base-catalyzed intramolecular attack of the amide group onto the ester bond at the 3'-end of the ODN (Scheme 6) (Aviñó et al., 1996). Thus, it is possible that different steric or inductive effects related to the nature of base might influence the reactivity of the oxalyl group. In our specific case, ease of cleavage of the oxalyl group with DBU followed the order $\mathrm{G}>\mathrm{A}>\mathrm{T}>\mathrm{C}$. At the 5'-end, a similar base-dependent reactivity has been observed for DMT groups towards acid cleavage. Indeed, it is well known that the lability of the DMT groups towards acid 
cleavage follows the order $\mathrm{G}>\mathrm{A}>>\mathrm{T}=\mathrm{C}$ (Atkinson and Smith, 1984). In order to improve the yields, the hydroquinone-O,O-diacetic acid (Q-linker) (Pon and Yu, 1997) was investigated. This linker is resistant to DBU solutions but cleavable with very mild nucleophiles. Unfortunately, following treatment with DBU, a brief exposure with a mild source of fluoride ions (i.e. triethylamine trihydrofluoride in $\mathrm{N}$-methylpyrrolidone or DMSO) failed.

\section{Melting experiments.}

The base-pairing properties of 5-azacytosine were measured on a duplex formed by two complementary pentadecamers in which ZCyt was paired with the four natural bases (Table 3). As expected, ZCyt formed the strongest base pair with G, although the Z:G base pair is $9{ }^{\circ} \mathrm{C}$ less stable than the natural $\mathrm{C}$ : $\mathrm{G}$ base pair. Mismatches of $\mathrm{Z}$ are also less stable than $\mathrm{C}$ mismatches (ca. $3{ }^{\circ} \mathrm{C}$ ) except for $\mathrm{Z}$ : $\mathrm{C}$, which is as stable as the $\mathrm{C}$ : $\mathrm{C}$ mismatch. The strong decrease in melting temperature observed for the Z:G base pair could be due to the 5-azacytosine ring being involved in rapid equilibrium between syn and anti conformations in which the former rotamer is unable to hydrogen bond effectively with $\mathrm{G}$ via the carbonyl group at C-2. It is also likely that if the ODN is heated to $90{ }^{\circ} \mathrm{C}$ during the preparation of the duplex the ZCyt may undergo ring opening and loss of formate. In order to estimate the impact of this potential decomposition during heating, five consecutive melting curves were recorded. We observed that the melting curve reproduced well after each heating cycle but with a small decrease in melting temperature of approximately $1{ }^{\circ} \mathrm{C}$ per cycle. Thus, we can conclude that a small decomposition of ZCyt may occur during each cycle and that the melting temperatures reported in Table 3 may be at least one degree lower than the real melting temperature.

\section{Conclusions}

In this paper, we describe the preparation of the phosphoramidite derivative of 5aza-2'-deoxycytidine protected with the NPEOC group. This phosphoramidite allows the efficient preparation of ODNs containing ZCyt provided that mild, non-hydrolytic conditions are used during the removal of the NPE, NPEOC groups. The extreme lability of the $\mathrm{Z}$ interferes not only with the preparation of the protected phosphoramidite and the removal of the protecting groups, but also with the purification of the modified oligonucleotides. In spite of these drawbacks, we have successfully prepared several ODNs containing one or more ZCyt units that have the appropriate length and purity 
required for biological studies. A report studying the inhibitory properties of these ZCytmodified oligonucleotides against DNA methyltransferase has been submitted (Brank et al., submitted). We have observed that yields of ODNs are dependent on the nature of the nucleoside present at the 3 '-end, probably due to a lower efficiency in the cleavage from the ODN-support linkage. Therefore, a good alternative for a future investigation is the use of photolabile linkers (Greenberg and Gilmore, 1994). The results presented here are of special interest for the preparation of ODNs carrying sensitive molecules such as mutagenic or reactive bases (Aviñó et al., 1995), or labile internucleotide bonds (Vivès et el., 1999).

\section{ACKNOWLEDGEMENTS}

We are thankful to Dr. Matthias Wilm and Gitta Neubauer for obtaining mass spectra.

\section{REFERENCES}

ALUL, R.H., SINGMAN, C.N., ZHAN, G. and LETSINGER, R.L.(1991) Oxalyl-CPG: A labile support for the synthesis of sensitive oligonucleotide derivatives. Nucleic Acids Res., 19, 1527-1532.

ATKINSON, T., and SMITH, M. (1984). Solid-phase synthesis of oligodeoxyribonucleotides by the phosphite-triester. In: Oligonucleotide synthesis. A practical approach. M.J. Gait, ed. (IRL Press, Oxford), pp. 35-81.

AVIÑÓ, A, and ERITJA, R. (1994) Use of Npe-protecting groups for the preparation of oligonucleotides without using nucleophiles during the final deprotection. Nucleosides \& Nucleotides, 13, 2059-2069.

AVIÑÓ, A., GÜIMIL-GARCIA, R., MARQUEZ, V.E., and ERITJA, R. (1995) Preparation and properties of oligodeoxynucleotides containing 4-O-butylthymine, 2fluorohypoxanthine and 5-azacytosine. Bioorg. Med. Chem. Lett., 5, 2331-2336. 
AVIÑÓ, A., GÜIMIL GARCÍA, R., DÍAZ, A., ALBERICIO, F., and ERITJA, R. (1996) A comparative study of supports for the synthesis of oligonucleotides without using ammonia. Nucleosides \& Nucleotides, 15, 1871-1889.

BEISLER, J.A. (1978) Isolation, characterization, and properties of a labile hydrolysis product of the antitumor nucleoside 5-azacytidine. J. Med. Chem., 21, 204.

BENDER, C.M., PAO, M.M., and JONES, P.A. (1998). Inhibition of DNA methylation by 5-aza-2'-deoxycytidine suppresses the growth of tumer cell lines. Cancer Res., 58, 95-101.

BRANK, A.S., ERITJA, R., GÜIMIL GARCÍA, R., MARQUEZ, V., and CHRISTMAN, J.K. (2001). Inhibition of HhaI DNA (Cytosine-C5) methyltransferase by oligodeoxyribonucleotides containing 5-aza-2'-deoxycytidine: Examination of the intertwined roles of co-factor, target transition state structure and enzyme conformation (submitted for publication).

CHRISTMAN, J.K. (1984) DNA methylation in friend erythroleukemia cells: the effects of chemically induced differentiation and of treatment with inhibitors of DNA methylation. Curr. Top. Microbiol. Immunol., 108, 49-78.

CHRISTMAN, J.K., SCHNEIDERMAN, N., and ACS, G. (1985) Formation of a highly stable complexes between 5-azacytosine-substituted DNA and specific non-histone nuclear proteins. Implications for 5-azacytidine-mediated effects on DNA methylation and gene expression. J. Biol. Chem., 260, 4059-4068.

CREUSOT, F., ACS, G., and CHRISTMAN, J.K. (1982) Inhibition of DNA methyltransferase and induction of friend erythroleukemia cell differentiation by 5azacytidine and 5-aza-2'-deoxycytidine. J. Biol. Chem., 257, 2041-2048.

DÍAZ, A.R., ERITJA, R., and GÜIMIL GARCÍA, R., (1997) Synthesis of oligodeoxynucleotides containing 2-substituted guanine derivatives using 2-fluoro-2'deoxyinosine as common nucleoside precursor. Nucleosides \& Nucleotides, 16, 20352051. 
ERITJA, R., ROBLES, J., AVIÑÓ, A., ALBERICIO, F., and PEDROSO, E. (1992) A synthetic procedure for the preparation of oligonucleotides without using ammonia and its application for the synthesis of oligonucleotides containing O-4-alkyl thymidines. Tetrahedron, 48, 4171-4182.

ERITJA, R., MARQUEZ, V.E., and GÜIMIL GARCÍA, R.(1997) Synthesis and properties of oligonucleotides containing 5-aza-2'-deoxycytidine. Nucleosides \& Nucleotides 16, 1111-1114.

FERRER, E., FÀBREGA, C., GÜIMIL GARCÍA, R., AZORÍN, F. and ERITJA, R. (1996) Preparation of oligonucleotides containing 5-bromouracil and 5-methylcytidine. Nucleosides \& Nucleotides, 15, 907-921.

GODDARD, A.J., and MARQUEZ, V.E. (1988) Synthesis of 2'-deoxy-5,6-dihydro-5azacytosine. Its potential application in the synthesis of DNA containing dihydro-5-aza and 5-azacytosine bases. Tetrahedron Lett., 29, 1767-1770.

GODDARD, A.J., and MARQUEZ, V.E. (1989) Synthesis of 2'-deoxy-5,6-dihydro-5azacytosine and 5-azacytosine at specific CpG sites. Nucleosides \& Nucleotides, 8, 1015-1018.

GOLDBERG, J., GRYN, J., RAZA, A., BENNETT, J., BROWMAN, G., BRYANT, J., and GRUNWALD, H. (1993) Mitoxantrone and 5-azacytidine for refractory/relapsed anII or cmI in blast crisis: A leukemia intergroup study. Am., J. Hematol., 43, 286-290.

GREENBERG, M.M. and GILMORE, J.L. (1994) Cleavage of oligonucleotides from solid-phase supports using o-nitrobenzyl photochemistry. J. Org. Chem., 59, 746-753.

HIMMELSBACH, F., SCHULZ, B.S., TRICHTINGER, T. CHARUBALA, R., and PFLEIDERER, W. (1984) The p-nitrophenylethyl (NPE) group. A versatile new blocking group for phosphate and aglycone protection in nucleosides and nucleotides. Tetrahedron, 40, 59-72. 
LIN, K.T., MOMPARLER, R.L., and RIVARD, G.H. (1981) High-performance liquid chromatographic analysis of chemical stability of 5-aza-2'deoxycytidine. J. Pharm. Sci., 70, 1228-1232.

MARQUEZ, V.E., GODDARD, A., ALVAREZ, E., FORD, H., CHRISTMAN, J.K., SHEIKHNEJAD, G., BRANK, A., MARASCO, C.J., SUFFRIN, J.R., O’GARA, M., and CHENG, X. (1999) Oligonucleotides containing 5,6-dihydro-5-azacytosine at CpG sites can produce potent inhibition of DNA cytosine-C5-methyltransferase without covalently binding to the enzyme. Antisense \& Nucleic Acid drug Development, 9, 415421.

MOMPARLER. R.L., COTE, S., and ELIOPOULOS, N. (1997) Pharmacological approach for optimization of the dose schedule of 5-aza-2'-deoxycytidine (Decitabine) for the therapy of leukemia. Leukemia, 11, 175-180.

PINTO, A., and ZAGONEL, V. (1993) 5-Aza-2'-deoxycytidine (decitabine) and 5azacytidine in the treatment of acute myeloid leukemias and myelodysplastic syndromes: Past, present and future trends. Leukemia, 7 Suppl 1, 51-60.

PISKALA, A., and SORM, F. (1978) 4-amino-1- $\beta$-D-ribofuranosyl-s-triazin-2(1H)-one (5-azacytidine). In Nucleic Acid Chemistry, Improved and New Synthetic Procedures, Methods and Techniques. Part one. L.B. Townsend and R.S. Tipson, eds, (John Wiley \& Sons, New York), pp. 443-449.

PON, R.T. and YU, S. (1997) Hydroquinone-O,O'-diacetic acid ('Q-linker') as a replacement for succinyl and oxalyl linker arms in solid phase oligonucleotide synthesis. Nucleic Acids Res., 25, 3629-3635.

ROCHETTE, J., CRAIG, J.E., and THEIN, S.L. (1994) Fetal hemoglobin levels in adults. Blood Rev., 8, 213-224.

SAMBROOK, J., FRITSCH, E.F., and MANIATIS, T. (1980) In Molecular cloning a laboratory manual. Cold-Spring Harbor Laboratory Press, New York, pp.10.59-10.61. 
SCHEIDT, K.A., CHEN, H., FOLLOWS, B.C., CHEMLER, S.R., COFFEY, D.S., and ROUSH, W.R. (1998) Tris (dimethylamino)sulfonium difluorotrimethylsilicate, a mild reagent for the removal of silicon protecting groups. J. Org. Chem., 63, 6436-6437.

SHEIKHNEJAD, G., BRANK, A., CHRISTMAN, J.K., GODDARD, A., ALVAREZ, E., FORD, H. Jr., MARQUEZ, V.E., MARASCO, C.J., SUFRIN, J.R., O’GARA, M., and CHENG, X. (1999) Mechanism of inhibition of DNA (Cytosine-C5)methyltransferases by oligodeoxyribonucleotides containing 5,6-dihydro-5-azacytosine. J. Mol. Biol., 285, 2021-2034.

TI, G.S., GAFFNEY, B.L. and JONES, P.A. (1982). Transient protection: Efficient oneflask syntheses of protected deoxynucleotides. J. Am. Chem. Soc., 104, 1316-1319.

VIVÈS, E., DELL’AQUILA, C., BOLOGNA, J.C., MORVAN, F., RAYNER, B., and IMBACH, J.L. (1999) Lipophillic pro-oligonucleotides are rapidly and efficiently internalized in HeLa cells. Nucleic Acids Res., 27, 4071-4076. 
Figure and scheme legends.

Scheme 1. The NPEOC and the NPE protecting groups.

Scheme 2. Mechanism of removal of the NPEOC protecting group by non-nucleophilic bases.

Scheme 3. Preparation of the phosphoramidite of 5-aza-2'-deoxycytidine. DMT: dimethoxytrityl, NPEOC: 2-(p-nitrophenyl)ethoxycarbonyl, TMS: trimethylsilyl.

Scheme 4. Preparation and structures of the phosphoramidites and solid supports used in this work.

Scheme 5. Proposed pathway for the decomposition of 5-azacytosine in DNA as described for the free nucleoside (Beisler 1978; Lin et al., 1981).

Scheme 6. Proposed mechanism for the cleavage of the oxalyl linker by 1,8diazabicyclo[5.4.0]undec-7-ene (DBU). CPG: controlled pore glass.

FIG 1. Effect of ammonia and acetic acid on the integrity of ODNs containing ZCyt. Reactions containing 0.1 OD units of ODN III were incubated overnight with concentrated ammonia at room temperature (Lane 2) and at $55^{\circ} \mathrm{C}$ (Lane 3), and with $80 \%$ acetic acid (1hour) at room temperature (Lane 4). Following these treatments, the samples were concentrated to dryness. Samples were analyzed on a $20 \%$ acrylamide / $7 \mathrm{M}$ urea gel. Bands were visualized with ethidium bromide and UV-shadowing (Lane 4 did not stain well with ethidium bromide). Lane 1 show the migration of untreated ODN III and Lane 3 show the migration of a control ODN which has the same sequence than ODN III but containing cytosine instead of ZCyt.

FIG 2. 8M Urea-20\% polyacrylamide gel electrophoretic analysis of ZCyt- and CytODNs after exposure to $80 \%$ acetic acid at $37{ }^{\circ} \mathrm{C}$ for $60 \mathrm{~min}$. Lane 1 , ss-ZCyt-ODN (control-no treatment); Lane 2, ss-ZCyt-ODN (acid treated); Lane 3, ds-ZCyt (acid treated); Lane 4, ss-Cyt-ODN (acid treated); Lane 5, ds-Cyt-ODN (acid treated); Lane 6, ss-Cyt-ODN and Lane 7, ds-Cyt-ODN incubated with HhaI. Cleavage with this 
restriction endonuclease yields a radiolabeled fragment 8 bases long (arrow). A radiolabeled scission product 5' to ZCyt would be 6 bases long. Experimental details are presented in Material and Methods. 
Table 1: 5-Azacytosine (Z) ODNs prepared using two-HPLC steps (DMT-on and DMToff).

\begin{tabular}{|l|l|l|}
\hline Sequences (5'->3') & $\begin{array}{l}\text { OD units after first } \\
\text { HPLC (DMT-on) }\end{array}$ & $\begin{array}{l}\text { OD units after } \\
\text { second HPLC } \\
\text { (DMT-off) }\end{array}$ \\
\hline I TAGZTGA & 2.5 & 0.9 \\
\hline II TAZGZTGA & 6.3 & 2 \\
\hline III GCAATGGAZCCTCTA & 15 & 6 \\
\hline IV ATTGCGCATTCCGGATCZGCGATC & 25 & 7.5 \\
\hline V ATTGCGCATTCCGGATCCGZGATC & 2.3 & 0.8 \\
\hline
\end{tabular}


Table 2: ODN sequences having 5-azacytosine prepared in this work using one-step purification by HPLC ( $\mathrm{Z}=5$-azacytosine, $\mathrm{M}=5$-methylcytosine).

\begin{tabular}{|l|l|}
\hline Sequence (5' $->$ ' ${ }^{\prime}$ ) & OD units after HPLC \\
\hline III GCAATGGAZCCTCTA & 15 \\
\hline V ATTGCGCATTCCGGATCCGZGATC & 10 \\
\hline VI ATTGZCCATTCCGGATCCGCGATC & 8 \\
\hline VII ATTGCCCATTCZGGATCCGCGATC & 14 \\
\hline VIII ATTGZCCATTCZGATCZGZGATC & 5 \\
\hline IX ATTGCCCATTZCGGATCCGCGATC & 9 \\
\hline X TGTCAGZGCATGG & $24,35,45,42,21$ \\
\hline XI TGTCAGCGZATGG & 27 \\
\hline XII TGTCAGZGCATGGATGGTTATAAT & 15 \\
\hline XIII ATTGCCMATTCCGGATCZGCGATC & 3 \\
\hline XIVATGAGCMAAAAAAAAAAZGGCTCA & 45 \\
\hline XV ATGAGZMAAAAAAAAAACGGCTCA & 55 \\
\hline
\end{tabular}


Table 3. Melting temperatures $\left({ }^{\circ} \mathrm{C}\right)$ of duplexes containing 5-aza-2'-deoxycytidine (ZCyt) base pairs (0.15 M NaCl, 0.05 M Tris-HCl buffer, $\mathrm{pH} 7.5$ )

\section{5' GCAATGGAXCCTCTA 3' \\ 3’ CGTTACCTYGGAGAT5'}

\begin{tabular}{|l|l|l|}
\hline & $X=Z$ & $X=C$ \\
\hline$Y=G$ & 50 & 59 \\
\hline$Y=A$ & 44 & 47 \\
\hline$Y=C$ & 45 & 45 \\
\hline$Y=T$ & 43 & 46 \\
\hline
\end{tabular}




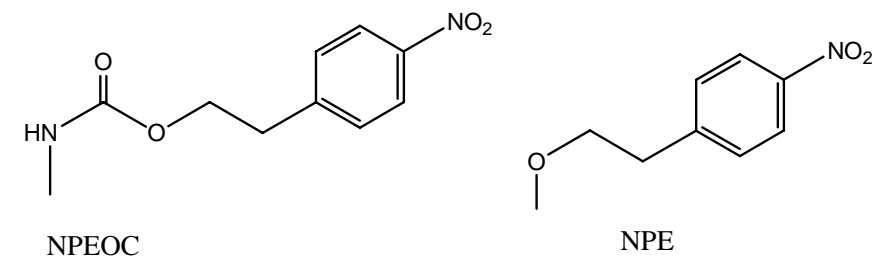

Scheme 1 


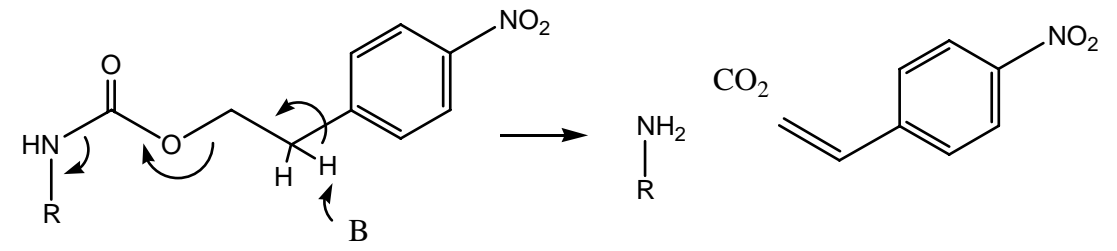

Scheme 2 


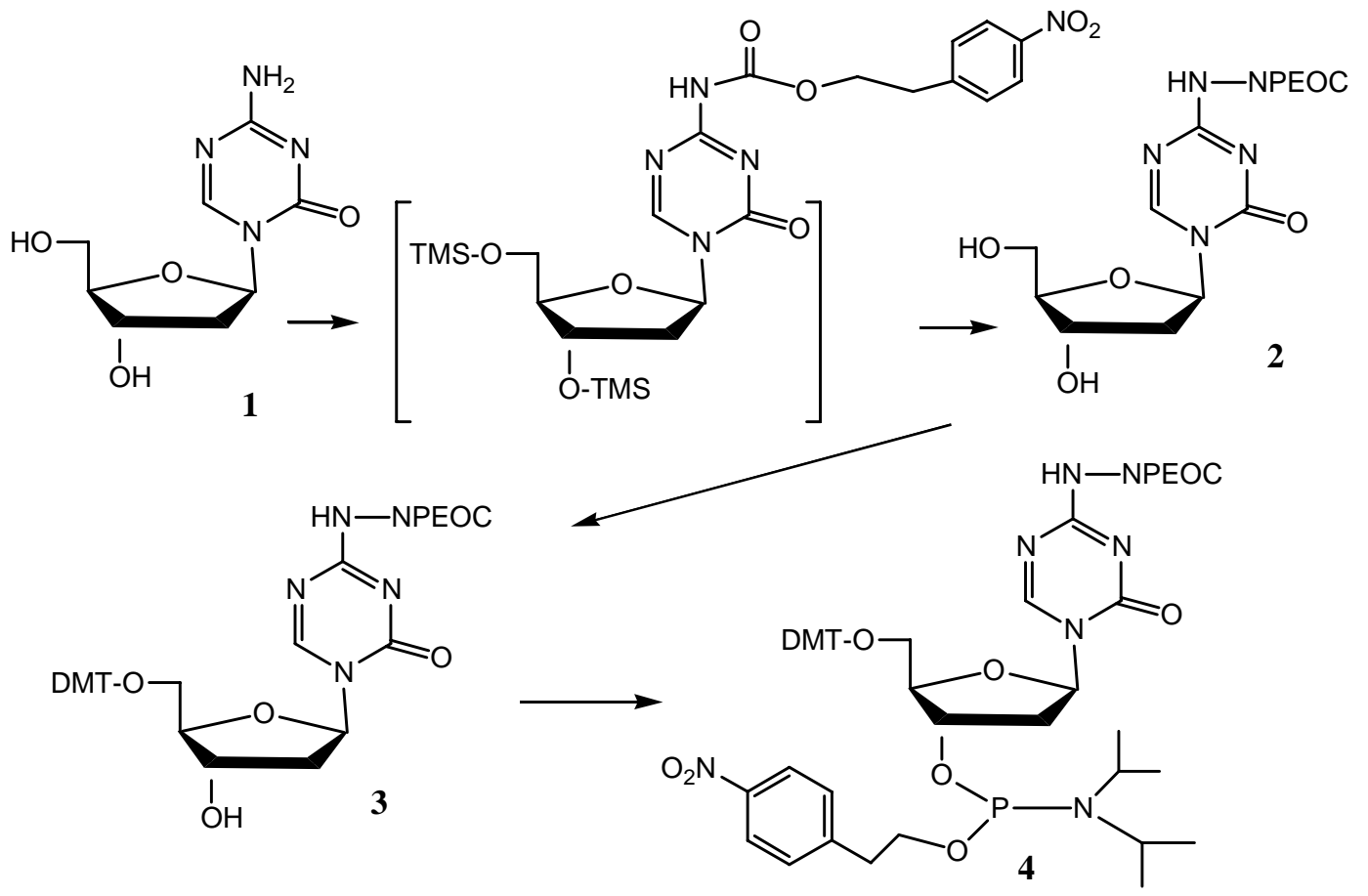

Scheme 3 


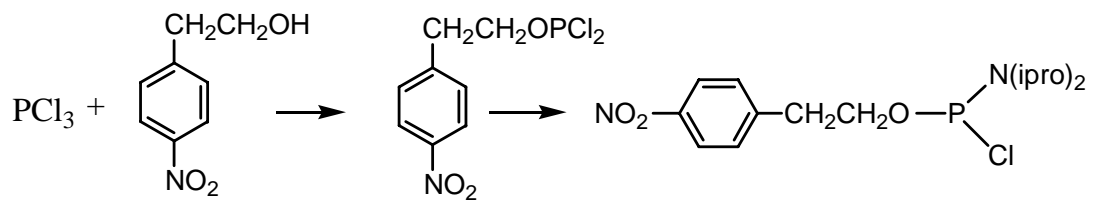<smiles>[R16]OCC1OC([B])CC1O</smiles><smiles></smiles><smiles>[B]C1CC(OC(=O)C(=O)NC(=O)OCc2ccccc2)C(CO[Na])O1</smiles><smiles>[B]=NC(=O)OCCc1ccc([N+](=O)[O-])cc1</smiles><smiles>Cn1cnc2c(OCCc3ccc([N+](=O)[O-])cc3)nc(NC(=O)OCCNC(=O)O[Na])nc21</smiles><smiles>Cc1cn(C)c(=O)nc1NC=O</smiles>

Scheme 4 


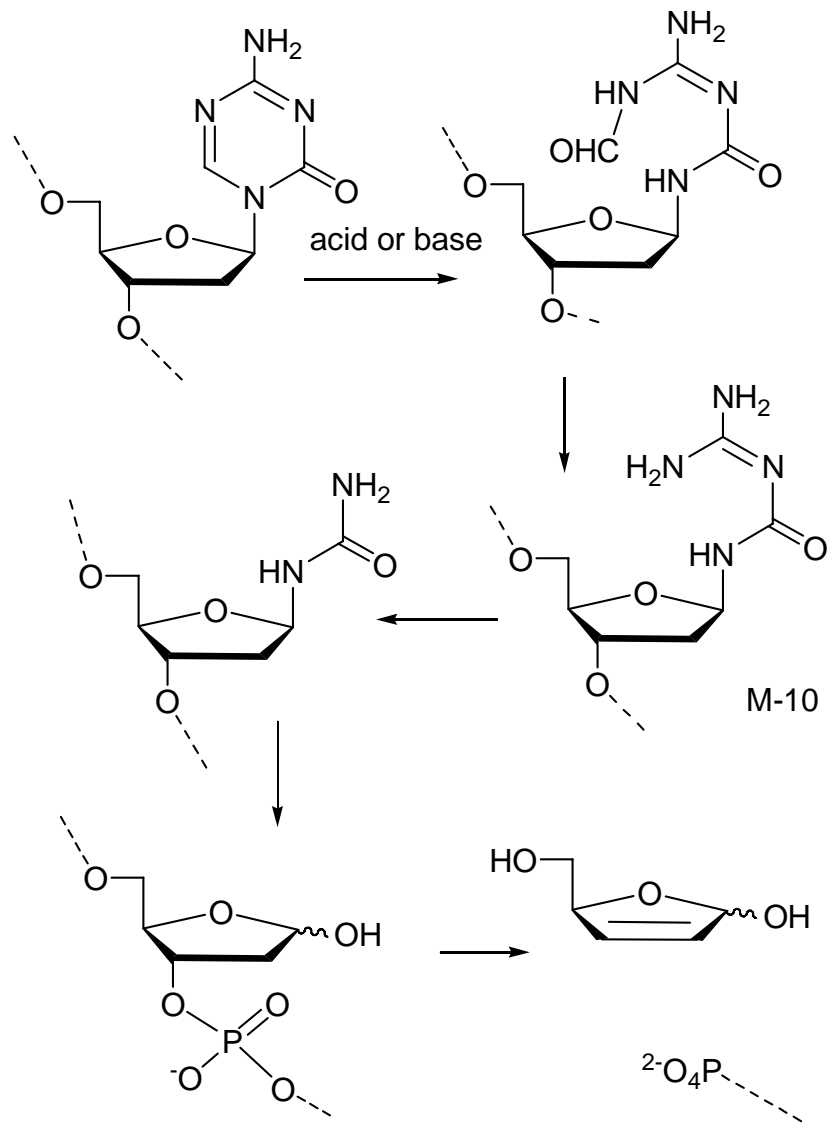

scheme 5 


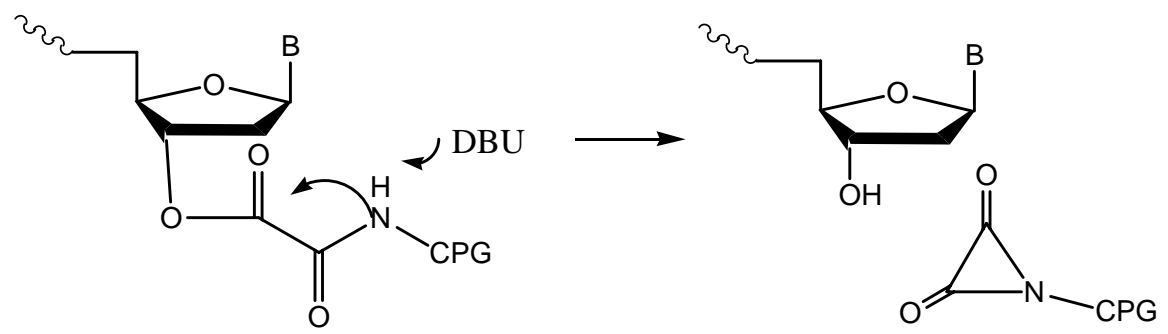

Scheme 6 
$\begin{array}{lllll}1 & 2 & 3 & 4 & 5\end{array}$

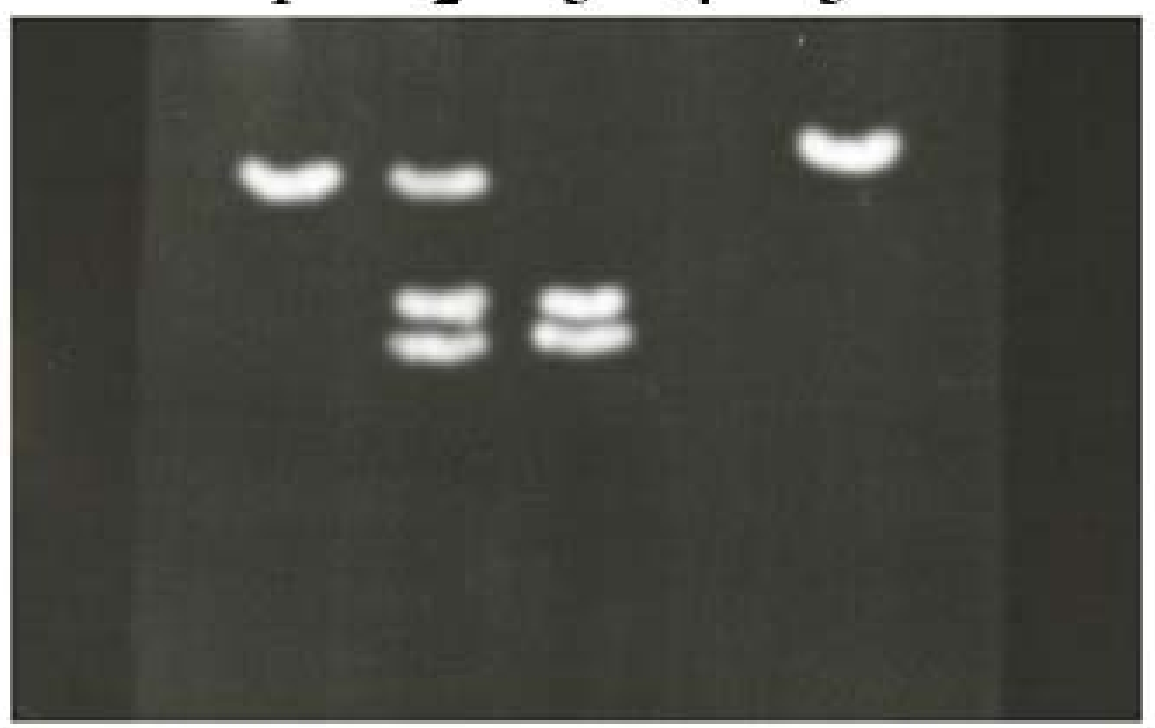

FIG. 1 


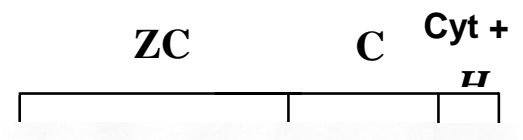

$\begin{array}{llllll}1 & 2 & 3 & 4 & 5 & 6\end{array}$

Fig.2 